\title{
Assistência de enfermagem na saúde da mulher frente ao câncer do colo do útero: revisão integrativa
}

\author{
Nursing care in women's health with cervical cancer: integrative review \\ Atención de enfermería en la salud de la mujer con cáncer de cuello uterino: revisión integradora
}

Recebido: 31/10/2021 | Revisado: 08/11/2021 |Aceito: 11/11/2021 | Publicado: 20/11/2021

Welmer Danilo Rodrigues Rocha
ORCID: https://orcid.org/0000-0003-4495-2436
E-mail: welmerrocha@gmail.com
Instituto Federal de Educação, Ciência e Tecologia do Tocantins, Brasil
Alexia Mariana da Silva Nogueira
ORCID: https://orcid.org/0000-0002-6449-4789
Faculdade de Colinas do Tocantins, Brasil
E-mail: alexiamariana320@gmail.com
Anna Laura Almeida de Araújo
ORCID: https://orcid.org/0000-0003-1052-8856
Faculdade de Colinas do Tocantins, Brasil
E-mail: annalaura13.al@gmail.com
Kamilla Germana da Silva
ORCID: https://orcid.org/0000-0002-8464-2013
Faculdade de Colinas do Tocantins, Brasil
E-mail: kamillager.s@gmail.com
Kyaren Sena da Silva Sousa
ORCID: https://orcid.org/0000-0001-7936-3311
Faculdade de Colinas do Tocantins, Brasil
E-mail: kyarensena7@gmail.com

\begin{abstract}
Resumo
A enfermagem é um grande pilar atuando no atendimento da saúde da mulher, tendo contribuição fundamental na educação em saúde e na realização da consulta de enfermagem para prevenção e manejo do câncer do colo do útero. Diante deste exposto, o presente estudo objetivou analisar as ações da assistência de enfermagem na prevenção e manejo do câncer do colo do útero, com base em uma revisão da literatura. Na realização da pesquisa, utilizou-se uma revisão integrativa da literatura, sobre o tema proposto, com abordagem qualitativa e exploratória. Os estudos científicos foram pesquisados nas principais bases de dados utilizadas na área da ciência em saúde (LILACS, Medline, SciElo e PubMed) e a seleção dos trabalhos iniciou-se por uma leitura prévia do resumo, a fim de verificar a associação do trabalho com o tema proposto. Após uma extensa pesquisa bibliográfica foram selecionados 16 artigos que melhor atenderam ao objetivo proposto. A análise crítica das informações copiladas dos trabalhos amostrados, possibilitou a identificação das principais significações em relação os desafios da enfermagem perante o diagnóstico do câncer do colo do útero; o papel da enfermagem frente a prevenção do câncer do colo do útero; e as estratégias de enfermagem como ação eficaz na prevenção do câncer do colo do útero. O papel do enfermeiro na promoção da saúde e nos comportamentos de prevenção de doenças é muito importante, uma vez que têm impacto nas atividades de promoção da saúde dos pacientes.
\end{abstract}

Palavras-chave: Promoção de saúde; Câncer cervical; Consulta de enfermagem; Saúde da mulher.

\begin{abstract}
Nursing is a great pillar acting in women's health care, having a fundamental contribution in health education and in carrying out nursing consultations for the prevention and management of cervical cancer. Given this, this study aimed to analyze the actions of nursing care in the prevention and management of cervical cancer, based on a literature review. In carrying out the research, an integrative literature review was used, on the proposed topic, with a qualitative and exploratory approach. Scientific studies were searched in the main databases used around health science (LILACS, Medline, SciElo and PubMed) and the selection of works started with a previous reading of the abstract, to verify the association of the work with the proposed theme. After an extensive literature search, 16 articles that best met the proposed objective were selected. The critical analysis of the information collected from the sampled works made it possible to identify the main meanings in relation to the challenges faced by nursing in the diagnosis of cervical cancer; the role of nursing in the prevention of cervical cancer; and nursing strategies as an effective action to prevent cervical cancer. The role of nurses in health promotion and disease prevention behaviors is very important, as they have an impact on patients' health promotion activities.
\end{abstract}

Keywords: Health promotion; Cervical cancer; Nursing consultation; Women's health. 


\section{Resumen}

La enfermería es un gran pilar que actúa en el cuidado de la salud de la mujer, teniendo un aporte fundamental en la educación para la salud y en la realización de las consultas de enfermería para la prevención y manejo del cáncer cervicouterino. Ante esto, este estudio tuvo como objetivo analizar las acciones de los cuidados de enfermería en la prevención y manejo del cáncer de cuello uterino, a partir de una revisión de la literatura. Para la realización de la investigación se utilizó una revisión bibliográfica integradora, sobre el tema propuesto, con un enfoque cualitativo y exploratorio. Se realizaron búsquedas de estudios científicos en las principales bases de datos utilizadas en el área de las ciencias de la salud (LILACS, Medline, SciElo y PubMed) y la selección de trabajos se inició con la lectura previa del resumen, con el fin de verificar la asociación del trabajo con la temática propuesta. Luego de una extensa búsqueda bibliográfica, se seleccionaron los 16 artículos que mejor cumplieron con el objetivo propuesto. El análisis crítico de la información recolectada de los trabajos muestreados permitió identificar los principales significados en relación a los desafíos que enfrenta la enfermería en el diagnóstico del cáncer cervicouterino; el papel de la enfermería en la prevención del cáncer cervicouterino; y estrategias de enfermería como acción eficaz para prevenir el cáncer de cuello uterino. El papel de las enfermeras en las conductas de promoción de la salud y prevención de enfermedades es muy importante, ya que tienen un impacto en las actividades de promoción de la salud de los pacientes.

Palabras clave: Promoción de la salud; Cáncer de cuello uterino; Consulta de enfermería; La salud de la mujer.

\section{Introdução}

O câncer do colo do útero (CCU), conhecido também como câncer de cérvix ou cervical, é o terceiro tipo de câncer de maior frequência no Brasil, sendo o mais comum entre mulheres, segundo o Instituto Nacional do Câncer (Inca, 2019). Apresenta cerca de 15.590 novos casos a cada ano, sendo considerado um problema de saúde pública (Ribeiro et al., 2019). É uma doença que ocorre lentamente, onde o precursor para o seu desenvolvimento é a infecção causada pelo Papilomavírus Humano (HPV) (INCA, 2021a). Apesar da causa principal, outros fatores são colaboradores para a sua evolução, estando entre eles tabagismo, início precoce da atividade sexual e histórico familiar (Inca, 2021b).

A melhor forma de reduzir essa doença é através do diagnóstico prematuro (Brasil, 2013). No Brasil, o diagnóstico é realizado através do exame citopatológico, mais conhecido como Papanicolau, um exame fácil e acessível que tem sido a melhor maneira para o rastreamento do CCU (Brasil, 2020). O mecanismo para realização do Papanicolau é confiável e seguro para diminuição das taxas de morbimortalidade por esse fator, visto que sendo identificado com antecedência há chances de 100\% de cura (Aoyama et al., 2019).

De acordo com Instituto Nacional do Câncer, o exame citopatológico Papanicolau deve ser realizado logo após o início da vida sexual, e é indicado mesmo quando não há sintomas aparentes, principalmente em mulheres na faixa etária de 25 a 64 anos (Inca, 2011). É de suma relevância a orientação sobre a consulta periódica e o conhecimento acerca do CCU, além da importância de incentivar a população do exame preventivo para rastreamento do câncer do colo do útero (Leite et al., 2020).

Embora seja reconhecida a relevância desse exame, diversos estudos apresentam os principais fatores que interferem na rejeição das mulheres em não realizar o exame de prevenção, entre eles estão: idade avançada, questões culturais, sentimentos de vergonha, medo de sentir dor e a falta de conhecimento sobre a importância da realização do exame (Ferreira, 2009; Gurgel et al., 2019). Com o intuito de fortalecer as políticas de saúde para a mulher, o Ministério da Saúde lançou o Programa de Assistência Integral à Saúde da Mulher (PAISM), o qual vem promovendo importantes avanços na promoção da saúde da mulher (Brasil, 2004).

A fim de garantir a efetividade dos rastreamentos, atualmente o Programa Nacional de Combate ao Câncer do Colo do Útero (PNCC) continua a promover exames citopatológicos, contando com níveis primário, secundário e terciário como o principal método de rastreamento para o CCU (Inca, 2021a). Esta é uma estratégia abordada principalmente na atenção primária, da qual a enfermagem possui relevante papel para a prevenção e controle do CCU (Teixeira, et al., 2017).

Neste âmbito, a enfermagem é um grande pilar atuando no atendimento da saúde da mulher, tendo contribuição fundamental na utilização de ferramentas como a educação em saúde e a realização da consulta de enfermagem na prevenção e manejo do CCU (Backes et al., 2012). Deste modo, a orientação através da educação em saúde possibilita contribuir para 
adesão de mulheres na realização do Papanicolau. Assim como, o diagnóstico precoce impede a evolução e permite um manejo satisfatório com um bom prognóstico dessa doença (OPAS, 2016).

Diante do exposto, a questão norteadora do presente estudo consiste em investigar quais estratégias são utilizadas pela enfermagem como ferramenta para promover a prevenção e redução da taxa de morbimortalidade por CCU? Para responder este questionamento, objetivou-se analisar as ações da assistência de enfermagem na prevenção e manejo do câncer do colo do útero, com base em uma revisão da literatura.

\section{Metodologia}

Utilizou-se uma revisão integrativa da literatura, sobre o tema proposto, com abordagem qualitativa e exploratória. Para fins de compreensão, Galvão e Pereira (2014) definem a revisão integrativa como um método muito empregado na área da saúde, usando comumente a compilação de dados para obter resultados. Sendo, um estudo secundário que se baseia nos estudos primários realizados.

Os estudos científicos foram pesquisados nas bases de dados: LILACS (Literatura Latino-Americana e do Caribe em Ciências da Saúde), Medline (Medical Literature Analysis and Retrieval System Online), SciElo (Scientific Electronic Library Online) e PubMed (US National Library of Medicine), com a combinação dos seguintes descritores: câncer do colo do útero "and" ações de enfermagem "and" prevenção.

A seleção dos trabalhos iniciou-se por uma leitura prévia do resumo, a fim de verificar a associação do trabalho com o tema proposto. Os critérios de inclusão definidos para a seleção dos artigos foram: artigos inteiramente disponíveis relacionados ao objeto de pesquisa, artigos disponíveis em idiomas português e inglês, artigos que fossem indexados nas bases de dados, artigos publicados no período de 2015 a 2021. Foram excluídas do portfólio amostral, trabalhos que consistiam em formato de teses, dissertações e monografias. Foram excluídos também estudos duplicados, resenhas e trabalhos editoriais.

A pesquisa foi realizada pelo acesso on-line, no período de agosto a outubro de 2021. Inicialmente, foram obtidos 50 artigos, após a leitura, e posteriormente selecionados conforme os critérios de inclusão e exclusão, totalizando dezesseis (16) artigos que compuseram a amostra, como demostrado na (Figura 1).

Figura 1. Fluxograma mostrando o processo de seleção dos artigos incluídos na revisão integrativa sobre a assistência de enfermagem na saúde da mulher frente ao câncer do colo do útero.

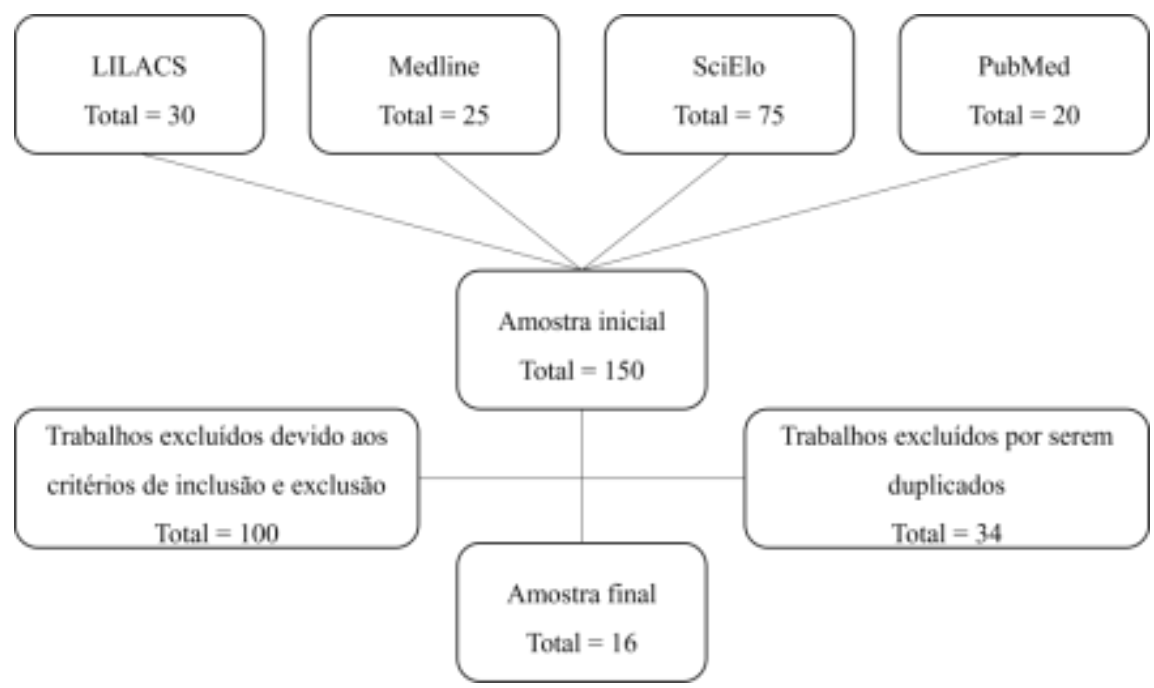

Fonte: Autores (2021). 


\section{Resultados e Discussão}

A partir da revisão integrativa, utilizando-se os descritores e as bases de dados mencionados, foram selecionados 16 artigos que melhor atenderam ao objetivo proposto na presente pesquisa. No Quadro 1 é descrito as características de cada estudo exposto, sintetizando-se os autores, ano de publicação, título, objetivo geral e desfecho dos estudos.

Quadro 1. Síntese da produção científica sobre a assistência de enfermagem na saúde da mulher frente ao câncer do colo do útero, no período de 2015 a 2021.

\begin{tabular}{|c|c|c|c|}
\hline Autores/Ano & Título & Objetivo & Desfecho \\
\hline $\begin{array}{c}\text { Costa et al., } \\
2017\end{array}$ & $\begin{array}{l}\text { Os desafios do enfermeiro } \\
\text { perante a prevenção do } \\
\text { câncer do colo do útero }\end{array}$ & $\begin{array}{l}\text { Conscientizar o uso do exame } \\
\text { citopatológico como método de } \\
\text { prevenção e relatar as dificuldades } \\
\text { que o enfermeiro enfrenta para } \\
\text { realizar coleta e do que ele dispõe } \\
\text { para melhorar a adesão da população } \\
\text { feminina. }\end{array}$ & $\begin{array}{l}\text { O enfermeiro deve orientar adequadamente as } \\
\text { mulheres acerca dos benefícios da prevenção, } \\
\text { organizar a assistência preventiva, criar método } \\
\text { eficaz na abordagem da população feminina e } \\
\text { desenvolver estratégias que superem dificuldades } \\
\text { existentes, no intuito de diminuir a prevalência } \\
\text { desta neoplasia. }\end{array}$ \\
\hline $\begin{array}{c}\text { Souza e } \\
\text { Costa, } 2015\end{array}$ & $\begin{array}{l}\text { Conhecimento } r \text { de } \\
\text { mulheres sobre HPV e } \\
\text { câncer do colo do útero } \\
\text { após consulta de } \\
\text { enfermagem }\end{array}$ & $\begin{array}{l}\text { Compreender a capacidade de } \\
\text { assimilação das mulheres que } \\
\text { realizam o exame Papanicolau } \\
\text { acerca do papilomavírus humano e } \\
\text { sua relação com o câncer do colo do } \\
\text { útero. }\end{array}$ & $\begin{array}{l}\text { Este estudo mostrou a persistência do } \\
\text { desconhecimento de mulheres sobre o } \\
\text { papilomavírus humano e sua relação com o câncer } \\
\text { do colo uterino, após a consulta de enfermagem na } \\
\text { ESF para prevenção desse tipo de câncer. }\end{array}$ \\
\hline $\begin{array}{l}\text { Leite et al., } \\
\quad 2018\end{array}$ & $\begin{array}{l}\text { Exame Papanicolau: } \\
\text { fatores que influenciam a } \\
\text { não realização do exame } \\
\text { em mulheres de } 40 \text { a } 65 \\
\text { anos }\end{array}$ & $\begin{array}{l}\text { Caracterizar os fatores que } \\
\text { influenciam mulheres de } 40 \text { a } 65 \\
\text { anos de idade a não realizarem o } \\
\text { exame Papanicolau. }\end{array}$ & $\begin{array}{l}\text { O medo do diagnóstico e a vergonha são os } \\
\text { principais fatores que influenciam a não adesão das } \\
\text { mulheres ao exame Papanicolau, na faixa etária } \\
\text { estudada. }\end{array}$ \\
\hline $\begin{array}{c}\text { Santos e } \\
\text { Varela, } 2015\end{array}$ & $\begin{array}{l}\text { Prevenção do câncer do } \\
\text { colo uterino: motivos que } \\
\text { influenciam a não } \\
\text { realização do exame de } \\
\text { Papanicolau }\end{array}$ & $\begin{array}{l}\text { er a importância do exame } \\
\text { vo e os motivos que levam } \\
\text { mulheres a não realizarem o }\end{array}$ & $\begin{array}{l}\text { Os resultados mostram que a falta de adesão ao } \\
\text { preventivo pela população feminina deve-se a } \\
\text { fatores como o desconhecimento do câncer uterino, } \\
\text { do exame e da sua realização, medo e vergonha e } \\
\text { outros de ordem pessoal. }\end{array}$ \\
\hline $\begin{array}{l}\text { Azevedo et } \\
\text { al., } 2016\end{array}$ & $\begin{array}{l}\text { Fatores que influenciam a } \\
\text { não realização do exame } \\
\text { de Papanicolau e o } \\
\text { impacto de ações } \\
\text { educativas }\end{array}$ & $\begin{array}{l}\text { Identificar as causas da não } \\
\text { realização do exame de Papanicolau, } \\
\text { como também a aplicação de } \\
\text { palestras educativas, visando } \\
\text { observar se havia mudança na } \\
\text { percepção acerca do exame } \\
\text { citopatológico. }\end{array}$ & $\begin{array}{l}\text { Os principais motivos que levam à não realização } \\
\text { do exame de Papanicolau são medo, vergonha e } \\
\text { falta de tempo, sendo as ações educativas de suma } \\
\text { importância, pois através delas pode-se alterar a } \\
\text { percepção em relação às formas de prevenção do } \\
\text { câncer do colo uterino e da frequência de } \\
\text { realização do exame de Papanicolau. }\end{array}$ \\
\hline $\begin{array}{c}\text { Sousa et al., } \\
2017\end{array}$ & $\begin{array}{lr}\text { Caracterização } & \text { das } \\
\text { alterações citopatológicas } & \\
\text { e fatores de riscos } \\
\text { associados } & \text { ao } \\
\text { desenvolvimento } & \text { do } \\
\text { câncer de colo útero } & \end{array}$ & $\begin{array}{l}\text { Identificar na literatura a } \\
\text { caracterização das alterações } \\
\text { citopatológicas e fatores de riscos } \\
\text { associados ao desenvolvimento do } \\
\text { câncer de colo uterino, descrevendo } \\
\text { o conhecimento produzido na } \\
\text { temática. }\end{array}$ & $\begin{array}{l}\text { A realização do exame citopatológico é a atividade } \\
\text { indispensável na detecção precoce de alterações } \\
\text { que possam levar ao desenvolvimento do câncer de } \\
\text { colo de útero. Com medidas simples, rápida, eficaz } \\
\text { e de baixo custo. }\end{array}$ \\
\hline $\begin{array}{r}\text { Nób } \\
\text { al., }\end{array}$ & $\begin{array}{l}\text { Importância da assistência } \\
\text { de enfermagem na } \\
\text { realização do exame } \\
\text { citopatológico: um olhar } \\
\text { bibliográfico }\end{array}$ & $\begin{array}{llll}\text { Averiguar a } & \text { importância da } \\
\text { assistência de enfermagem na } & \text { de } \\
\text { realização do exame citopatológico. }\end{array}$ & $\begin{array}{l}\text { O exame é considerado um método de excelência } \\
\text { para diagnosticar alterações na cérvice uterina } \\
\text { podendo reduzir de forma significativa sua } \\
\text { incidência, tendo o enfermeiro papel prioritário, } \\
\text { realizando a busca ativa de mulheres que já } \\
\text { iniciaram a vida sexual e enfatizando durante as } \\
\text { consultas a importância da realização periódica do } \\
\text { exame Papanicolau para a prevenção do câncer de } \\
\text { colo de útero. }\end{array}$ \\
\hline $\begin{array}{c}\text { Hanna et al., } \\
2020\end{array}$ & $\begin{array}{l}\text { Mortality due to cancer } \\
\text { treatment delay: } \\
\text { systematic review and } \\
\text { meta-analysis }\end{array}$ & $\begin{array}{l}\text { Quantificar a associação de atraso no } \\
\text { tratamento do câncer e mortalidade } \\
\text { para cada aumento de quatro } \\
\text { semanas no atraso para informar as } \\
\text { vias de tratamento do câncer. }\end{array}$ & $\begin{array}{l}\text { A demora no tratamento do câncer é um problema } \\
\text { nos sistemas de saúde em todo o mundo. Mesmo } \\
\text { um atraso de quatro semanas no tratamento do } \\
\text { câncer, este, por sua vez, está associado ao } \\
\text { aumento da mortalidade nas indicações cirúrgicas, } \\
\text { de tratamento sistêmico e de radioterapia para sete }\end{array}$ \\
\hline
\end{tabular}




\begin{tabular}{|c|c|c|c|}
\hline & & & tipos de câncer, incluindo o do colo do útero. \\
\hline $\begin{array}{c}\text { Andrades, } \\
2018\end{array}$ & $\begin{array}{l}\text { A atuação do enfermeiro } \\
\text { na orientação e prevenção } \\
\text { do câncer do colo do útero } \\
\text { na atenção básica }\end{array}$ & $\begin{array}{l}\text { Identificar e descrever a importância } \\
\text { das ações e orientações realizadas } \\
\text { pelo enfermeiro na prevenção do } \\
\text { câncer do colo do útero na Atenção } \\
\text { básica. }\end{array}$ & $\begin{array}{l}\text { Evidenciou-se que o enfermeiro é primordial na } \\
\text { prevenção ao controle do câncer do colo do útero e } \\
\text { suas ações e orientações são elementos } \\
\text { fundamentais na Atenção básica. }\end{array}$ \\
\hline $\begin{array}{l}\text { Amaral et } \\
\text { al., } 2017\end{array}$ & $\begin{array}{l}\text { Prevenção do câncer de } \\
\text { colo de útero: a atuação do } \\
\text { profissional enfermeiro } \\
\text { nas unidades básicas de } \\
\text { saúde }\end{array}$ & $\begin{array}{l}\text { Analisar a importância do } \\
\text { enfermeiro na prevenção do câncer } \\
\text { de colo do útero e sua atuação } \\
\text { profissional no contexto da estratégia } \\
\text { de saúde da família. }\end{array}$ & $\begin{array}{l}\text { O profissional enfermeiro é o principal responsável } \\
\text { dentro da atenção primária na prevenção desta } \\
\text { doença por ser capaz de analisar as dificuldades } \\
\text { encontradas para a realização do exame } \\
\text { citopatológico. }\end{array}$ \\
\hline $\begin{array}{c}\text { Santos et al., } \\
2019\end{array}$ & $\begin{array}{l}\text { Educação em saúde como } \\
\text { estratégia de prevenção do } \\
\text { câncer do colo do útero: } \\
\text { um enfoque na atuação do } \\
\text { enfermeiro }\end{array}$ & $\begin{array}{l}\text { Analisar a produção científica sobre } \\
\text { as estratégias utilizadas para } \\
\text { prevenção do câncer do colo do } \\
\text { útero por enfermeiros na atenção } \\
\text { básica. }\end{array}$ & $\begin{array}{l}\text { O enfermeiro tem papel fundamental no processo } \\
\text { informativo, incentivo, preventivo e assistencial às } \\
\text { mulheres com câncer de colo uterino, sendo } \\
\text { necessário evidenciar uma boa qualificação e } \\
\text { cuidado ético dos profissionais. }\end{array}$ \\
\hline $\begin{array}{l}\text { Teixeira et } \\
\text { al., } 2017\end{array}$ & $\begin{array}{ll}\text { Atuação do enfermeiro da } \\
\text { Atenção Primária no } \\
\text { controle do câncer de } \\
\text { mama }\end{array}$ & $\begin{array}{l}\text { Analisar as ações realizadas por } \\
\text { enfermeiros da Atenção Primária em } \\
\text { Saúde para rastreamento } \\
\text { oportunístico do câncer de mama, } \\
\text { tendo como parâmetro a proposta do } \\
\text { Ministério da Saúde. }\end{array}$ & $\begin{array}{l}\text { Os enfermeiros têm realizado ações para o controle } \\
\text { do câncer de mama, mas existem algumas não } \\
\text { conformidades entre as ações executadas e as } \\
\text { propostas do Ministério da Saúde para o } \\
\text { rastreamento desta neoplasia. }\end{array}$ \\
\hline $\begin{array}{c}\text { Musa et al., } \\
2017\end{array}$ & $\begin{array}{l}\text { Effect of cervical cancer } \\
\text { education and provider } \\
\text { recommendation for } \\
\text { screening on screening } \\
\text { rates: A systematic review } \\
\text { and meta-analysis }\end{array}$ & $\begin{array}{l}\text { O objetivo desta revisão sistemática } \\
\text { foi compreender as evidências do } \\
\text { efeito da educação sobre o câncer do } \\
\text { colo do útero em comparação com as } \\
\text { condições de controle nas taxas de } \\
\text { rastreamento do câncer na população } \\
\text { de mulheres em risco. }\end{array}$ & $\begin{array}{l}\text { As descobertas do estudo apoiam a implementação } \\
\text { de intervenções educacionais sobre o câncer do } \\
\text { colo do útero baseadas em teorias para aumentar a } \\
\text { participação das mulheres em programas de } \\
\text { rastreamento, principalmente quando se trata de } \\
\text { comunidades com baixos níveis de alfabetização. }\end{array}$ \\
\hline $\begin{array}{l}\text { Silva et al., } \\
\quad 2017\end{array}$ & $\begin{array}{l}\text { Prevenção do câncer } \\
\text { cérvico-uterino: uma ação } \\
\text { realizada pelos } \\
\text { enfermeiros da estratégia } \\
\text { saúde da família? }\end{array}$ & $\begin{array}{l}\text { Analisar as intervenções de } \\
\text { prevenção e promoção da saúde } \\
\text { relacionadas a detecção precoce do } \\
\text { câncer cérvico-uterino desenvolvidas } \\
\text { pelos enfermeiros das equipes da } \\
\text { Estratégia Saúde da Família (ESF). }\end{array}$ & $\begin{array}{l}\text { Faz-se necessário que as ações dos enfermeiros da } \\
\text { ESF, no que concerne a detecção precoce e } \\
\text { rastreamento do câncer do colo do útero sejam } \\
\text { redirecionadas e, ademais, que a educação em } \\
\text { saúde possa ser implantada de forma a promover o } \\
\text { empoderamento das mulheres com vistas ao } \\
\text { autocuidado e a promoção da saúde. }\end{array}$ \\
\hline $\begin{array}{c}\text { Souza e } \\
\text { Alves, } 2015\end{array}$ & $\begin{array}{l}\text { Estratégias educativas para } \\
\text { prevenção e redução da } \\
\text { morbimortalidade } \\
\text { câncer do colo uterino }\end{array}$ & $\begin{array}{l}\text { Analisar as estratégias educativas } \\
\text { utilizadas pelo enfermeiro para a } \\
\text { prevenção e redução de } \\
\text { morbimortalidade do câncer de colo } \\
\text { uterino no Brasil, buscando } \\
\text { evidências na literatura sobre as } \\
\text { estratégias educativas eficazes na } \\
\text { prevenção do câncer de colo uterino. }\end{array}$ & $\begin{array}{l}\text { As estratégias educativas utilizadas pelo } \\
\text { enfermeiro para a prevenção de câncer do colo } \\
\text { uterino são largamente diversificadas e necessitam } \\
\text { ser combinadas entre si, pois, considerando-se que } \\
\text { não há uma metodologia única e específica, elas } \\
\text { devem ser adaptadas conforme as características } \\
\text { loco-regionais do público-alvo. }\end{array}$ \\
\hline $\begin{array}{c}\text { Souza et al., } \\
2018\end{array}$ & $\begin{array}{l}\text { Câncer de colo de útero: o } \\
\text { papel do enfermeiro na } \\
\text { prevenção no âmbito da } \\
\text { estratégia da saúde da } \\
\text { família }\end{array}$ & $\begin{array}{l}\text { Descrever, por meio de uma revisão } \\
\text { de literatura, o papel do enfermeiro } \\
\text { quanto a prevenção do câncer do } \\
\text { colo do útero em âmbito da } \\
\text { estratégia da saúde da família. }\end{array}$ & $\begin{array}{l}\text { O enfermeiro na atenção primaria de saúde tem } \\
\text { papel importante nas ações de promoção da saúde } \\
\text { e de prevenção do câncer do colo do útero pela sua } \\
\text { atuação diretamente junto às mulheres, sendo que, } \\
\text { é esse profissional que realiza o preparo, a coleta } \\
\text { do material para o exame, atividades de promoção } \\
\text { a saúde e o acompanhamento dessas mulheres. }\end{array}$ \\
\hline
\end{tabular}

Fonte: Autores (2021).

Após a seleção dos artigos e análise crítica das informações em foco, foi identificado as principais significações em relação os desafios da enfermagem perante o diagnóstico do câncer do colo do útero; o papel da enfermagem frente a prevenção do câncer do colo do útero; e as estratégias de enfermagem como ação eficaz na prevenção do câncer do colo do útero. 


\subsection{Os desafios da enfermagem perante o diagnóstico do câncer do colo do útero}

Dentre todos os fatores de riscos, que dificultam a prevenção/diagnóstico do CCU, é possível citar a prática da não realização do exame citopatológico (EC) como o principal. Neste sentido, Costa et al. (2017) realizaram um estudo com o objetivo de conscientizar o uso do EC como método de prevenção e concluíram que, para efetivar o EC, é recomendado que o enfermeiro oriente adequadamente as mulheres acerca dos benefícios da prevenção, criando métodos eficazes na abordagem da população feminina e desenvolvendo estratégias que superem dificuldades existentes, como a não realização do exame, no intuito de diminuir a prevalência desta neoplasia.

A não realização do EC pode estar relacionado a sentimentos como medo, vergonha, ansiedade, timidez, comodismo, descuido, além das crenças, tabus, e a falta de conhecimento a respeito das doenças sexualmente transmissíveis (DST). Reforçando esta discussão, Souza e Costa (2015) apontam em seu estudo uma deficiência na comunicação entre o enfermeiro e paciente durante a consulta regular na ESF, o que persiste no desconhecimento das mulheres sobre o papilomavírus humano e sua relação com o CCU e, consequentemente, uma dificuldade na prevenção desse tipo de câncer.

Já os fatores relacionados ao profissional de enfermagem e/ou à unidade de saúde, que por muitas vezes tornam-se desafios perante o diagnóstico do PCCU, Costa et al. (2017) citam que o acesso ao serviço com dificuldade para agendamento do EC, às filas de espera, o pouco envolvimento dos profissionais da saúde e a falta de materiais para a coleta do exame são fatores reais e que impactam negativamente no diagnóstico precoce do CCU. Ademais, os autores supracitados ainda declaram que a falta de tempo do enfermeiro devido ao trabalho em excesso comprometendo a qualidade do serviço ofertado, inviabiliza o diagnóstico e a prevenção desta neoplasia.

Estes fatores atrelados tanto ao sentimento da paciente como ao profissional de enfermagem, que acarretam a não realização do EC, podem levar ao atraso do tratamento do câncer, quando este se fizer necessário. As consequências disso são adversas, mas a principal seria a mortalidade, desfecho este que poderia ser evitado em casos de um diagnóstico precoce e tratamento da neoplasia. Hanna et al. (2020) em seu estudo, que objetivaram quantificar a associação de atraso no tratamento do câncer e mortalidade para cada aumento de quatro semanas, observaram, por meio de uma meta-análise, que foi significativa a associação entre o atraso do tratamento e aumento da mortalidade. Este estudo demonstra que, mesmo um atraso de quatro semanas no tratamento, este pode ocasionar em um impacto negativou ou positivo no desfecho clínico da paciente.

Apesar das estratégias em saúde no âmbito nacional, para aumentar a cobertura do exame, ainda existem dificuldades relacionadas a realização do exame, influenciando na baixa cobertura a nível nacional e, consequentemente, torna-se um desafio perante o diagnóstico do CCU (Leite et al., 2018; Santos \& Varela, 2015). Portanto, conhecer os fatores que dificultam a realização do exame citopatológico é fundamental para traçar o perfil populacional das mulheres e dessa maneira possibilitar a criação de estratégias mais adequadas, a cada realidade, o que consequentemente venha a favorecer o rastreamento e diagnóstico precoce do CCU.

\subsection{O papel da enfermagem frente a prevenção do câncer do colo do útero}

A prevenção do CCU dá-se principalmente pela realização do exame citopatológico. No entanto, são relatados fatores que contribuem para a não realização deste exame, como já abordado no presente estudo. Deste modo, as ações educativas em saúde são de suma importância no enfrentamento desta doença, pois através delas pode-se alterar a percepção em relação às formas de prevenção do CCU e da frequência de realização do exame de Papanicolau. Azevedo et al. (2016) abordam esse aspecto com maestria em um estudo que objetivou identificar as causas da não realização do exame de Papanicolau. Sendo assim, os autores mencionados declararam que as ações educativas são de suma importância para minimizar a vergonha e falta de tempo que as pacientes declaram como justificativa para a não realização do EC, pois, através da educação em saúde podese alterar a percepção em relação às formas de prevenção do CCU e da frequência de realização do exame de Papanicolau. 
Outro estudo que reforça esta discussão, foi realizado por Musa et al. (2017), no qual objetivou, por meio de uma revisão sistemática, compreender as evidências do efeito da educação sobre o CCU em comparação com as condições de controle nas taxas de rastreamento do câncer na população de mulheres elegíveis em risco. Os autores mencionados declaram que, o uso de intervenções educacionais baseadas em teoria aumentou significativamente as taxas de diagnóstico para o CCU, em mais do que o dobro. Além disso, oferecer às mulheres a opção de auto amostragem para o teste do papilomavírus humano (HPV), aumentou, também, o rastreamento desta neoplasia, fortalecendo ainda mais a ideia de que intervenções educacionais são necessárias, especialmente quando direcionadas as comunidades com baixos níveis de alfabetização.

Pode-se destacar, também, o papel do enfermeiro frente a prevenção do CCU focado na atenção primária, uma vez que está é o ponto crucial para o controle da neoplasia em questão (Costa et al., 2017). Neste contexto, o enfermeiro realiza atividades técnicas, gerenciais e educacionais específicas no âmbito de sua competência e, por meio do contato com as usuárias/pacientes, concentram esforços para reduzir tabus, mitos e preconceitos em relação a prevenção do CCU, além de buscar a convicção da população feminina sobre as vantagens da prevenção (Costa et al., 2017).

Nóbrega et al. (2016) em estudo com o objetivo de averiguar a importância da assistência de enfermagem na realização do EC. O trabalho dos autores reforça o que tem sido discutido ao longo deste estudo e enfatizaram o papel do profissional de enfermagem como agente prioritário na redução significativa de incidência do CCU. Para tal, Sousa et al. (2017) determinam que o enfermeiro deve realizar uma busca ativa de mulheres que já iniciaram a vida sexual enfatizando, durante as consultas de enfermagem, a importância da realização periódica do EC para a prevenção do CCU.

Além da atenção à saúde feita nas unidades básicas de saúde, Andrades (2018) também aborda que a equipe profissional de enfermagem pode realizar visitas porta a porta e as consultas de enfermagem de forma integral e humanizada, orientando todos os procedimentos de coleta de CE, de forma a auxiliar as mulheres e fazer o encaminhamento adequado para mulheres com alterações citológicas. Neste âmbito, Santos et al. (2019) declararam em seu estudo que o profissional de enfermagem pode efetuar visitas em residências e consultas de enfermagem, de maneira integralizada e humanizada, norteando cada procedimento da coleta do EC para a prevenção do CCU.

A atuação do enfermeiro nas ações de promoção e prevenção do CCU é de extrema importância, pois suas atividades são realizadas em múltiplas dimensões, podendo-se destacar, segundo Santos et al. (2019): prática de consulta de enfermagem, ações educativas com equipes de saúde e a comunidade, gestão e prestação de recursos materiais e técnicos, controle de qualidade dos exames, pesquisas em saúde, comunicação e divulgação de resultados. É neste aspecto multifacetado que se estabelece o nexo necessário com a prática, e a partir da compreensão da existência local e da análise contínua dos resultados, a ação sistemática visa a redução dos danos causados pela doença (Amaral et al., 2017).

A atuação do enfermeiro, no geral, tem o intuito de ajudar o direcionamento das ações da enfermagem para a resolução ou minimização das necessidades individuais dos pacientes. Com isto, Nóbrega et al. (2016) afirmam que o enfermeiro contribui no reconhecimento precoce do processamento saúde-doença, realizando promoção, prevenção, recuperação e reabilitação do indivíduo, família e comunidade. Desta maneira, as ações de enfermagem ajudarão para um bom atendimento as mulheres da unidade básica de saúde, com encaminhamento adequado as pacientes que apresentarem alterações citológicas, além de passar informações necessárias a essa população, relacionada aos fatores de risco, trabalhando na prevenção e descoberta precoce do CCU (Amaral et al., 2017).

\subsection{Estratégias de enfermagem como ação eficaz na prevenção do câncer do colo do útero}

A principal estratégia de enfermagem, como ação eficaz na prevenção do CCU, pode-se citar a consulta em enfermagem, a qual é imprescindível no rastreamento e diagnóstico da neoplasia em questão, possibilitando uma prevenção de agravos na saúde da mulher. No entanto, ainda é necessária uma efetividade desta ação para controle do CCU, visto que 
Teixeira et al. (2017) afirmaram que existem algumas não conformidades entre as ações executadas e as propostas do Ministério da Saúde para o rastreamento desta neoplasia, o que pode ser abordado em estudos futuros enfatizando está temática.

É fundamental destacar a relevância que as ações preventivas e de detecção precoce concentrado na atenção básica e na atenção primária que se podem evitar o aparecimento da enfermidade, por meio das intervenções em seus fatores de ricos (Souza \& Alves, 2015). Por causa disso, deve-se contribuir para educação da população a respeito da utilização do preservativo e identificá-lo como um essencial instrumento de prevenção, já que a infecção do HPV possui papel relevante no progresso desta neoplasia; incentivar adoção de hábitos saudáveis, como alimentação adequadas e exercícios físico regulares, redução a exposição ao cigarro, correção das deficiências profissionais e incentivo a realização de exames preventivos (Andrades, 2018; Silva et al., 2017).

Outra ação, além da feita de forma educativa na própria unidade de saúde, é a feita fora das unidades de saúde, concretizadas através de palestras, rodas de conversas e orientações individuais a respeito da importância da prática de exames citopatológicos desde o início da vida sexual (Costa et al., 2017). Sendo assim, o enfermeiro pode contar com a ajuda dos agentes de saúde (ACS) para ficarem mais próximas da população e gerenciar melhor suas atividades, realizar enfoque sobre o CCU na sala de espera, ensinar profissionais sensibilizados para aconselhar as mulheres que estão na sala de espera a realizar o exame, marcar consultas por livre demanda, respeitando-se o limite de vagas de que cada profissional dispõe (Souza et al., 2018).

Neste sentido, para melhor aderir ao movimento preventivo do CCU, é necessário aproximar as ações por parte do profissional de enfermagem das crenças das mulheres assistidas, de tal forma que proporcione comportamentos preventivos de acordo com a cultura de cada mulher e seu ambiente (Amaral et al., 2017). Ademais, quanto mais abrangente for o programa de prevenção e mais atuante for o enfermeiro, melhor será o resultado dessas ações.

\section{Considerações Finais}

Os enfermeiros desempenham um papel importante na prevenção de doenças e podem ajudar os pacientes a obter mais informações sobre, melhorando assim a saúde dos seus pacientes. Estimular esses pacientes a procurar ajuda médica imediatamente em caso de alguma anormalidade, é uma dificuldade que o enfermeiro precisa reconhecer e enfrentar no seu dia a dia.

O papel do enfermeiro na promoção da saúde e nos comportamentos de prevenção de doenças é muito importante, uma vez que têm impacto nas atividades de promoção da saúde dos pacientes. Vale destacarmos a importância da consulta de enfermagem à pacientes, com foco no rastreamento do câncer do colo do útero, bem como as informações específicas prestadas nesta atividade de enfermagem, cuja prática torna-se uma valiosa oportunidade de prevenção do câncer do colo do útero.

Portanto, a efetuação da consulta de enfermagem ginecológica pode colaborar para maior abrangência do público-alvo na efetuação do exame citopatológico, sendo capaz de causar resultados em médio e longo períodos nos índices de morbimortalidade do câncer do colo do útero.

Sugere-se, contudo, que novos estudos sejam realizados visando a efetividade das ações de enfermagem na prevenção do câncer do colo do útero, tomando como base as propostas do Ministério da Saúde e demais órgãos responsáveis no enfrentamento desta neoplasia. Todavia, espera-se que esta pesquisa venha contribuir para estudos futuros, tornando a discussão acerca da Assistência de enfermagem na saúde da mulher frente ao câncer do colo do útero, algo mais robusto e consolidado no meio acadêmico e técnico/científico. 


\section{Referências}

Angelakis, I,. Austin, J. L., \& Gooding, P. (2020). Association of Childhood Maltreatment With Suicide Behaviors Among Young People A Amaral, M. S., Gonçalves, A. G. \& Silveira, L. C. G. (2017). Prevenção do câncer de colo de útero: a atuação do profissional enfermeiro nas unidades básicas de saúde. Revista Científica FacMais, 8(1), 197-223.

Andrades, N. B. (2018). A atuação do enfermeiro na orientação e prevenção do câncer do colo do útero na atenção básica. Cadernos Saúde e Desenvolvimento, $12(7), 5-26$.

Aoyama, E. A., Pimentel, A. S., Andrade, J. S., Daniel, W. V., Souza, R. A. G. \& Lemos, L. R. (2019). Assistência de enfermagem na prevenção do câncer de colo do útero. Brazilian Journal of Health Review, 2(1), 162-170.

Azevedo, A. G., Cavalcante, I. B., Cavalcante, J. B. \& Rolim, L. A. D. M. M. (2016). Fatores que influenciam a não realização do exame de Papanicolau e o impacto de ações educativas. Revista Brasileira de Análises Clínicas, 48(3), 253-257.

Backes, D. S., Backes, M. S., Erdmann, A. L. \& Büscher, A. (2012). O papel professional do enfermeiro no Sistema Único de Saúde: da saúde comunitária à estratégia de saúde da família. Ciência \& Saúde Coletiva, 17(1), 223-230.

Brasil. (2013). Controle dos cânceres do colo do útero e da mama. Ministério da Saúde, Secretaria de Atenção à Saúde, Departamento de Atenção Básica. $2^{a}$ ed. Brasília, DF: Editora do Ministério da Saúde, 124p.

Brasil. (2020). Papanicolau: exame preventivo de colo de útero. Ministério da Saúde. https://bvsms.saude.gov.br/papanicolau-exame-preventivo-de-colo-deutero/.

Brasil. (2004). Política Nacional de Atenção Integral à Saúde da Mulher: princípios e diretrizes. Brasília, DF: Ministério da Saúde.

Costa, F. K. M., Weigert, S. P., Burci, L. \& Nascimento, K. F. (2017). Os desafios do enfermeiro perante a prevenção do câncer do colo do útero. Revista Gestão \& Saúde, 17(1), 55-62.

Ferreira, M. L. S. M. (2009). Motivos que influenciam a não-realização do exame de Papanicolau segundo a percepção de mulheres. Escola Anna Nery Revista de Enfermagem, 13(2), 378-384.

Galvão, T. F. \& Pereira, M. G. (2014). Revisões sistemáticas da literatura: passos para sua elaboração. Epidemiologia e Serviços de Saúde, $23(1), 183-184$.

Gurgel, L. C., Sousa, A. A. S., Sousa, C. M. S., Brito, E. A. S., Leite, R. S. S., Santana, W. J. \& Vieira, P. D. (2019). Percepção de mulheres sobre o exame de prevenção de colo de útero Papanicolau: uma revisão integrativa da literatura. Revista Multidisciplinar e de Psicologia, 13(46), 434-445.

Hanna, T. P., King, W. D., Thibodeau, S., Paulin, G. A., O’Sulivan, D. E., Booth, C. M. \& Aggarwal, A. (2020). Mortality due to cancer treatment delay: systematic review and meta-analysis. The $B M J, 327,1-11$.

INCA. Detecção precoce. Instituto Nacional de Câncer José Alencar Gomes da Silva, 2021a. https://www.inca.gov.br/controle-do-cancer-do-colo-doutero/acoes-de-controle/deteccao-precoce.

INCA. Fatores de risco. Instituto Nacional de Câncer José Alencar Gomes da Silva, 2021b. https://www.inca.gov.br/controle-do-cancer-do-colo-doutero/fatores-de-risco.

INCA. Instituto Nacional de Câncer José Alencar Gomes da Silva. Diretrizes brasileiras para o rastreamento do câncer do colo do útero. Rio de Janeiro: INCA, 2011. 104p.

INCA. Instituto Nacional de Câncer José Alencar Gomes da Silva. Estimativa 2020: incidência de câncer no Brasil. Rio de Janeiro: INCA, 2019. 120p.

Leite, A. C., Silva, M. P. B., Alves, R. S. S., Feitosa, L. M. H., Ribeiro, R. N., Prado, A. M., Silva, L. S. B., Sousa, I. R. X., Fé, T. R. M., Oliveira, S. S. \& et al. (2020). Atribuições do enfermeiro no rastreamento do câncer de colo do útero em pacientes atendidas na Unidade Básica de Saúde. Research, Society and Development, 9, 11 .

Leite, K. N. S., Silva, J. P., Sousa, K. M., Rodrigues, S. C., Souza, T. A., Alves, J. P., Souza, A. R. D. \& Rodrigues, A. R. S. (2018). Exame Papanicolau: fatores que influenciam a não realização do exame em mulheres de 40 a 65 anos. Arquivos de Ciências da Saúde, 25(2), 15-19.

Musa, J., Achenbach, C. J., O’Dwyer, L. C. O., Evans, C. T., McHugh, M., Hou, L., Simon, M. A., Murphy, R. L. \& Jordan, N. (2017). Effect of cervical cancer education and provider recommendation for screening on screening rates: A systematic review and meta-analysis. PLoS One, 5(12), 1-28.

Nóbrega, A. L., Sousa, M. N. A., Sousa, K. M. O., Rodrigues, A. C. F. \& Oliveira, G. G. (2016). Importância da assistência de enfermagem na realização do exame citopatológico: um olhar bibliográfico. Temas em Saúde, 16(2), 81-104.

OPAS. Organização Pan-Americana da Saúde. Controle Integral do câncer do colo do útero: guia de práticas essenciais. Washington, DC: OPAS, 2016.

Ribeiro, A. M. N., Ribeiro, M. F. S., Costa, K. B., Oliveira, M. P. S., Lima, A. C.E., Cunha, M. A. P., Nascimento, I. C. S. \& Sotero, A. S. (2019). O papel do enfermeiro na prevenção do câncer do colo uterino. Brazilian Journal of Surgery and Clinical Research, 27(3), $132-134$.

Santos, A. C. S. \& Varela, C. D. S. (2015). Prevenção do câncer de colo uterino: motivos que influenciam a não realização do exame de Papanicolau. Revista Enfermagem Contemporânea, 4(2), 179-188.

Santos, J. S., Araújo, A. S., Milanêz, A. L. O., Moreno, A. N. V., Oliveira, B. A., Araújo, C. I. S., Morais, C. S., Araújo, E. A., Silva, E. P. O. \& Araújo, E. S. S. (2019). Educação em saúde como estratégia de prevenção do câncer do colo do útero: um enfoque na atuação do enfermeiro. Brazilian Journal of Surgery and Clinical Research, 27(1), 104-113. 
Research, Society and Development, v. 10, n. 15, e72101522606, 2021

(CC BY 4.0) | ISSN 2525-3409 | DOI: http://dx.doi.org/10.33448/rsd-v10i15.22606

Silva, A. B., Rodrigues, M. P., Oliveira, A. P. \& Melo, R. H. V. (2017). Prevenção do câncer cervicouterino: uma ação realizada pelos enfermeiros da estratégia saúde da família? Revista Ciência Plural, 3(2), 99-114.

Sousa, A. C. O., Costa, G. S., Reis, J. Q., Goiano, P. D. O. L. \& Calaça, M. B. (2017). Caracterização das alterações citopatológicas e fatores de riscos associados ao desenvolvimento do câncer de colo útero. Revista Uningá Review, 30(1), 67-71.

Souza, A. F. \& Costa, L. H. R. (2015). Conhecimento de mulheres sobre HPV e Câncer do colo do útero após consulta de enfermagem. Revista Brasileira de Cancerologia, 61(4), 343-350.

Souza, G. \& Alves, P. S. (2015). Estratégias educativas para prevenção e redução da morbimortalidade do câncer do colo uterino. Saúde e Pesquisa, 8(2), 317326.

Souza, S. M., Sobrinho, M. F. \& Gutierres, L. L. B. (2018). Câncer de colo do útero: o papel do enfermeiro na prevenção no âmbito da estratégia da saúde da família. Revista Saber Científico, 1(1), 1-7.

Teixeira, M. S., Goldman, R. E., Gonçalves, V. C. S., Gutiérrez, M. G. R. \& Figueiredo, E. N. (2017). Atuação do enfermeiro da Atenção Primária no controle do câncer de mama. Acta Paulista de Enfermagem, 30(1), 1-7. 